\title{
Rectangular group congruences on a semigroup
}

\author{
Roman S. Gigoń
}

Received: 13 September 2011 / Accepted: 1 August 2012 / Published online: 18 August 2012

(C) The Author(s) 2012. This article is published with open access at Springerlink.com

\begin{abstract}
We study rectangular group congruences on an arbitrary semigroup. Some of our results are an extension of the results obtained by Masat (Proc. Am. Math. Soc. 50:107-114, 1975). We show that each rectangular group congruence on a semigroup $S$ is the intersection of a group congruence and a matrix congruence and vice versa, and this expression is unique, when $S$ is $E$-inversive. Finally, we prove that every rectangular group congruence on an $E$-inversive semigroup is uniquely determined by its kernel and trace.
\end{abstract}

Keywords Rectangular group congruence $\cdot$ Group congruence $\cdot$ Matrix congruence

\section{Introduction and preliminaries}

A groupoid $S$ is called a left [right] zero semigroup if it satisfies the identity $x y=x$ $[x y=y]$. Further, by a rectangular band we shall mean the direct product of a left zero and a right zero semigroup. Moreover, a semigroup $S$ is said to be a rectangular group if it is isomorphic to the direct product $G \times M$ of a group $G$ and a rectangular band $M$.

Let $\mathcal{C}$ be a class of semigroups. We say that a congruence $\rho$ on a semigroup $S$ is a $\mathcal{C}$-congruence if $S / \rho \in \mathcal{C}$. For example, if $\mathcal{C}$ is the class of groups, then $\rho$ is called a group congruence on $S$ if $S / \rho$ is a group. In way of an exception, a congruence $\rho$ on a semigroup $S$ is said to be a matrix congruence if $S / \rho$ is a rectangular band. Note that every left [right] zero semigroup is a rectangular band, so every left [right] zero congruence on $S$ is a matrix congruence. Also, clearly the least matrix congruence

Communicated by Marcel Jackson.

R.S. Gigoń (凶)

Institute of Mathematics and Computer Science, Wroclaw University of Technology,

Wyb. Wyspianskiego 27, 50-370 Wroclaw, Poland

e-mail: romekgigon@tlen.pl 
on any semigroup $S$ exists. Denote it by $\psi$. Furthermore, every group congruence and every matrix congruence is a rectangular group congruence. Hence we say that a rectangular group congruence is proper if it is neither a group nor a matrix congruence. We first give necessary and sufficient conditions on a semigroup $S$ in order that it will have a proper rectangular group congruence. Furthermore, we show that every rectangular group congruence on $S$ is the intersection of a group congruence and a matrix congruence. In addition, if $S$ is $E$-inversive, then this expression is unique. Moreover, we prove that each rectangular group congruence on an $E$-inversive semigroup is uniquely determined by its kernel and trace. Before we start our study, we recall some definitions.

Let $S$ be a semigroup and $a \in S$. The set $W(a)=\{x \in S: x=x a x\}$ is called the set of all weak inverses of $a$ and so the elements of $W(a)$ will be called weak inverse elements of $a$. A semigroup $S$ is said to be E-inversive if for every $a \in S$ there exists $x \in S$ such that $a x \in E_{S}$, where $E_{S}$ (or briefly $E$ ) is the set of idempotents of $S$ (more generally, if $A \subseteq S$, then $E_{A}$ denotes the set of idempotents of $A$ ). It is easy to see that a semigroup $S$ is $E$-inversive if and only if $W(a)$ is nonempty for all $a \in S$. Hence if $S$ is $E$-inversive, then for every $a \in S$ there is $x \in S$ such that $a x, x a \in E_{S}$ [7,8]. Further, by $\operatorname{Reg}(S)$ we shall mean the set of regular elements of $S$ (an element $a$ of $S$ is called regular if $a \in a S a)$ and by $V(a)=\{x \in S: a=a x a, x=x a x\}$ the set of all inverse elements of $a$. It is well known that an element $a$ of $S$ is regular if and only if $V(a) \neq \emptyset$, so a semigroup $S$ is regular if and only if $V(a) \neq \emptyset$ for every $a \in S$. Finally, a regular semigroup $S$ is said to be orthodox if $E_{S}$ forms a subsemigroup of $S$.

The following result seems to belong to folklore.

Result 1.1 The following conditions concerning a semigroup $S$ are equivalent:

(i) $S$ is a rectangular band;

(ii) $S$ is nonwhere commutative, i.e., $\forall a, b \in S[a b=b a \Longrightarrow a=b]$;

(iii) $\forall a, b \in S[a b a=a]$;

(iv) $\forall a, b, c \in S\left[a^{2}=a, a b c=a c\right]$.

Recall from [9] that a nonempty subset $A$ of a semigroup $S$ is called left [right] dense if the condition $a b \in A$ implies that $a \in A[b \in A]$ for all $a, b \in S$. Further, $A$ is said to be quasi dense if the following two conditions hold:

(i) $\forall a \in S\left[a \in A \Longleftrightarrow a^{2} \in A\right]$;

(ii) $\forall a, b \in S[a b \in A \Longleftrightarrow a S b \subseteq A]$.

Finally, we say that $A$ is a quasi ideal of $S$ if $A S \cap S A \subseteq A$. For the connections between left [right] zero, matrix congruences on a semigroup $S$ and left dense right [right dense left] ideals, quasi dense subsemigroups of $S$ (respectively), the reader is referred to [9]. We note only some results of [9]. Firstly, denote by $X$ the set of all left dense right ideals of a semigroup $S$ and all right dense left ideals of a semigroup $S$ with the empty set included and $S$ excluded. Let $2^{X}$ be a family of all subsets of $X$ and $\mathcal{M C}(S)$ be the set of all matrix congruences on $S$. Define the map $\phi: 2^{X} \rightarrow \mathcal{M C}(S)$ by $\mathcal{X} \phi=\rho \mathcal{X}\left(\mathcal{X} \in 2^{X}\right)$, where

$$
\rho \mathcal{X}=\{(a, b) \in S \times S: \forall A \in \mathcal{X}[a, b \in A \text { or } a, b \notin A]\} .
$$


Result 1.2 (Theorem 5 [9]) The map $\phi$ is antitone (i.e., $\mathcal{X} \subseteq \mathcal{Y} \Longrightarrow \rho \mathcal{Y} \subseteq \rho \mathcal{X}$ ) and maps $2^{X}$ onto $\mathcal{M C}(S)$.

Result 1.3 (Corollary to Theorem 5 [9]) The relation $\rho_{X}$ is the least matrix congruence on a semigroup $S$. Moreover, we may replace (in the present result) the set $X$ by the set $Y$ of all quasi dense subsemigroups of $S$.

Result 1.4 (A part of Proposition 4, Theorem 9 [9]) The following conditions concerning a congruence $\rho$ on a semigroup $S$ are equivalent:

(i) $\rho$ is a matrix congruence on $S$;

(ii) every $\rho$-class of $S$ is a quasi dense subsemigroup of $S$;

(iii) every $\rho$-class of $S$ is a quasi ideal of $S$.

Conversely, a subsemigroup A of $S$ is quasi dense, when $A$ is a matrix of some $\psi$-classes of $S$. Thus $A$ is quasi dense if and only if $A$ is a $\rho$-class of some matrix congruence $\rho$ on $S$.

Result 1.5 (Theorem 14 [9]) Let $S$ be a matrix of semigroups $S_{l \lambda}$, where $\iota \in I$, $\lambda \in \Lambda$, such that every $S_{l \lambda}$ has an identity element $e_{l \lambda}$ and the set $M$ (say) of elements $e_{l \lambda}(l \in I, \lambda \in \Lambda)$ forms a subsemigroup of $S$. Then $M$ is a rectangular band. Further, $S_{l \lambda} \cong S_{J \mu}$ for all $\iota, j \in I, \lambda, \mu \in \Lambda$ and if we suppose that $1 \in I, \Lambda$, then $S$ is isomorphic to the direct product $M \times S_{11}$ of a rectangular band $M$ and $a$ semigroup $S_{11}$. Moreover, the semigroups $S_{l \lambda}$ are precisely the $\psi$-classes of $S$ and $S_{l \lambda}=e_{l \lambda} S_{l \lambda} e_{l \lambda}=e_{l \lambda} S e_{l \lambda}$ for all $\iota \in I, \lambda \in \Lambda$.

Notice that if $S$ is a rectangular group (that is, $S \cong M \times G$, where $M$ is a rectangular band and $G$ is a group), then we shall write rather $S=M \times G$ than $S \cong M \times G$. The following theorem is known but for example: Masat considered in [5, 6] a regular semigroup $S$ such that $E_{S}$ forms a rectangular band, and he did not know that $S$ is a rectangular group, and so we include a simple proof for the completeness. Green's relations on a semigroup $S$ are denoted by $\mathcal{L}^{S}, \mathcal{R}^{S}, \mathcal{H}^{S}, \mathcal{D}^{S}$ and $\mathcal{J}^{S}$. For undefined terms the reader is referred to the books $[3,4]$.

Theorem 1.6 The following conditions concerning a semigroup $S$ are equivalent:

(i) $S$ is a rectangular group;

(ii) $S$ is completely simple and orthodox;

(iii) $S$ is completely regular and satisfies the identity: $x^{-1} y y^{-1} x=x^{-1} x$;

(iv) $S$ is regular and $E_{S}$ forms a rectangular band.

Consequently, if $S$ is a rectangular group, then $S \cong E_{S} \times \mathcal{H}_{e}=E_{S} \times$ eSe for some (all) $e \in E_{S}$.

Proof (ii) $\Longrightarrow$ (i). If $S$ is completely simple, then $S$ is a matrix of groups $\mathcal{H}_{e}$ $\left(e \in E_{S}\right.$ ), since Lemma III.2.4 [4] implies that $\mathcal{H}$ is a matrix congruence on $S$, so $\mathcal{H}=\psi$. Clearly, every $\mathcal{H}_{e}$ has an identity element $e$. Also, (efe,e) $\in \mathcal{H}$ for all $e, f \in E_{S}$, that is, $e=e f e$ for all $e, f \in E_{S}$ (since $e f e \in E_{S}$ ). Hence $E_{S}$ is a rectangular band. Thus $S \cong E_{S} \times \mathcal{H}_{e}$ for some (all) $e \in E_{S}$ (by Result 1.5). 
(i) $\Longrightarrow$ (iii). Let $S=M \times G$, where $M$ is a rectangular band and $G$ is a group (with an identity 1). Define the mapping ${ }^{-1}: S \rightarrow S$ by $(a, g)^{-1}=\left(a, g^{-1}\right)$ for all $(a, g) \in S$. One can easily verify that $\left(S, \cdot,^{-1}\right)$ is completely regular, that is, $\left(x^{-1}\right)^{-1}=x, x x^{-1}=x^{-1} x$ and $x x^{-1} x=x$ for every $x \in S$. Further, suppose that $x=(a, g), y=(b, h) \in S$. Then

$$
x^{-1} y y^{-1} x=\left(a b^{2} a, 1\right)=\left(a^{2}, 1\right)=\left(a, g^{-1}\right)(a, g)=x^{-1} x .
$$

(iii) $\Longrightarrow$ (iv). Let $e \in E_{S}$. Since $e e^{-1}=e^{-1} e,\left(e^{-1}\right)^{-1}=e$, then

$$
e=e e^{-1} e=e^{-1} e=\left(e^{-1} e\right)^{2}=\left(e^{-1} e\right)\left(e e^{-1}\right)=e^{-1} e e^{-1}=e^{-1} \text {. }
$$

Hence $e^{-1} f f^{-1} e=e^{-1} e$, i.e., $e f e=e$ for all $e, f \in E_{S}$. Thus $E_{S}$ is a rectangular band. Consequently, the condition (iv) holds.

(iv) $\Longrightarrow$ (ii). Clearly, each idempotent of $S$ is primitive and $\mathcal{D}^{E_{S}}=E_{S} \times E_{S}$. Since $S$ is regular, then every element of $S$ is $\mathcal{D}$-related with some of its idempotent. It follows that $\mathcal{D}^{S}=\mathcal{J}^{S}=S \times S$. Thus $S$ is completely simple and orthodox.

By the trace $\operatorname{tr} \rho$ of a relation $\rho$ on a semigroup $S$ we shall mean the restriction of $\rho$ to the set $E_{S}$.

The following result will be useful in the proof of Theorems 2.2(iii), 2.5, 2.6.

Result 1.7 (Corollary 2.7 [1]) If $\rho$ is a matrix congruence on an E-inversive semigroup $S$, then every $\rho$-class of $S$ is $E$-inversive. Also, every matrix congruence on an E-inversive semigroup is uniquely determined by its trace.

Further, some preliminaries about group congruences on a semigroup $S$ are needed. A subset $A$ of $S$ is called (respectively) full; reflexive and dense if $E_{S} \subseteq A$; $\forall a, b \in S[a b \in A \Longrightarrow b a \in A]$ and $\forall s \in S \exists x, y \in S[s x, y s \in A]$. Also, define the closure operator $\omega$ on $S$ by $A \omega=\{s \in S: \exists a \in A[a s \in A]\}(A \subseteq S)$. We shall say that $A \subseteq S$ is closed (in $S$ ) if $A \omega=A$. Finally, a subsemigroup $N$ of a semigroup $S$ is said to be normal if it is full, dense, reflexive and closed (if $N$ is normal, then we shall write $N \triangleleft S$ ). Moreover, if a subsemigroup of $S$ is full, dense and reflexive, then it is called seminormal.

By the kernel $\operatorname{ker} \rho$ of a congruence $\rho$ on a semigroup $S$ we shall mean the set $\left\{x \in S:\left(x, x^{2}\right) \in \rho\right\}$.

The following two results follow directly from the definition of the group.

Lemma 1.8 Let $\rho$ be a group congruence on a semigroup $S$. Then $\operatorname{ker} \rho \triangleleft S$.

Lemma 1.9 Let $\rho_{1}, \rho_{2}$ be group congruences on a semigroup $S$. Then $\rho_{1} \subset \rho_{2}$ if and only if $\operatorname{ker} \rho_{1} \subset \operatorname{ker} \rho_{2}$.

Let $B$ be a nonempty subset of a semigroup $S$. Consider four relations on $S$ :

$$
\begin{aligned}
& \rho_{1, B}=\{(a, b) \in S \times S: \exists x \in S[a x, b x \in B]\} ; \\
& \rho_{2, B}=\{(a, b) \in S \times S: \exists x, y \in B[a x=y b]\} ; \\
& \rho_{3, B}=\{(a, b) \in S \times S: \exists x \in S[x a, x b \in B]\} ; \\
& \rho_{4, B}=\{(a, b) \in S \times S: \exists x, y \in B[x a=b y]\} .
\end{aligned}
$$


Lemma 1.10 [2] Let a subsemigroup $B$ of a semigroup $S$ be dense and reflexive. Then $\rho_{1, B}=\rho_{2, B}=\rho_{3, B}=\rho_{4, B}$.

If $B$ is a seminormal subsemigroup of $S$, then we denote the above four relations by $\rho_{B}$.

The following theorem says that there exists an inclusion-preserving bijection between the set of all normal subsemigroups of a semigroup $S$ and the set of all group congruences on $S$.

Theorem 1.11 [2] Let $B$ be a seminormal subsemigroup of a semigroup $S$. Then the relation $\rho_{B}$ is a group congruence on $S$. Moreover, $B \subseteq B \omega=\operatorname{ker} \rho_{B}$. If $B$ is normal, then $B=\operatorname{ker} \rho_{B}$.

Conversely, if $\rho$ is a group congruence on $S$, then there exists a normal subsemigroup $N$ of $S$ such that $\rho=\rho_{N}$ (in fact, $\left.N=\operatorname{ker} \rho\right)$. Thus there exists an inclusionpreserving bijection between the set of all normal subsemigroups of $S$ and the set of all group congruences on $S$.

Finally, the following remark will be useful.

Remark 1 Denote by $\sigma$ the least group congruence on a semigroup (if it exists). One can easily seen that if $S$ is an $E$-inversive semigroup (and so $E_{S}$ is dense), then there exists the least normal subsemigroup of $S$. In the light of the above theorem, every $E$-inversive semigroup possesses a least group congruence.

\section{Rectangular group congruences}

The following theorem gives necessary and sufficient conditions on a semigroup $S$ in order that it has a proper rectangular group congruence. (Notice that a normal subsemigroup $N$ of $S$ is called proper if $N \neq S$.)

Theorem 2.1 Let $S$ be a semigroup. The following conditions are equivalent:

(i) there exists a proper rectangular group congruence on $S$;

(ii) $S$ is a disjoint union of two or more quasi dense subsemigroups of $S$ and contains a proper normal subsemigroup of $S$;

(iii) there exists a non-universal group and a non-universal matrix congruence on $S$.

Proof (i) $\Longrightarrow$ (ii). Let $\rho$ be a proper rectangular group congruence on $S$, say $S / \rho$ is equal $M \times G$, where $M$ is a rectangular band, $G$ is a group (with identity 1 ). Note that $M \cong E_{M \times G}=\{(m, 1): m \in M\}$. Further, for all $m \in M$, define $Q_{m}$ to be the preimage of $\{m\} \times G$ by the canonical epimorphism $\rho^{\natural}$ from $S$ onto $S / \rho$. It follows easily from Result 1.1(iv) that $\{m\} \times G$ is a quasi dense subsemigroup of $M \times G$. Thus the preimage of $\{m\} \times G$ by $\rho^{\natural}$ is also such a subsemigroup of $S$ (by $M \cong E_{M \times G}$ ). Since $\rho$ is not a group congruence, then $|M|>1$, and so $S$ has a proper matrix congruence (Result 1.3). Hence $S$ is a disjoint union of two or more quasi dense subsemigroups of $S$, see Result 1.4 (notice that $S=\bigcup\left\{Q_{m}: m \in M\right\}$, where the union is disjoint, and 
this decomposition of $S$ induced, by the first part of Result 1.4, a matrix congruence on $S$ ). Let $N=\operatorname{ker} \rho$. Then $a \in N$ if and only if $a \rho \in E_{M \times G}$, that is, if and only if $a \rho \in M \times\{1\}$. Clearly, $N$ is a full subsemigroup of $S$. Also, $M \times\{1\}$ is reflexive in $M \times G$, so $N$ is reflexive in $S$. Furthermore, $N$ is dense, since $S / \rho$ is $E$-inversive. Finally, $N$ is closed in $S$, since $M \times\{1\}$ is closed in $M \times G$. Consequently, $N$ is a normal subsemigroup of $S$ and since $S / \rho$ is not a rectangular band, then $N$ is proper.

(ii) $\Longrightarrow$ (iii). This follows from Result 1.4 and Theorem 1.11.

(iii) $\Longrightarrow$ (i). Let $\rho_{1}$ be a non-universal matrix congruence on $S$ and $\rho_{2}$ be a nonuniversal group congruence on $S$. We show that $\rho=\rho_{1} \cap \rho_{2}$ is a proper rectangular group congruence on a semigroup $S$. Indeed, let $a \in S$. Since $S / \rho_{2}$ is regular, then $(a x a, a) \in \rho_{2}$ for some $x \in S$, so $(a x a, a) \in \rho$. Therefore $S / \rho$ is regular. Clearly, $x \rho_{2}$, where $x \in \operatorname{ker} \rho_{2}$ is an identity of the group $S / \rho_{2}$ and so $(x y x, x) \in \rho_{2}$ for all $x, y \in \operatorname{ker} \rho_{2}$. Hence $(x y x, x) \in \rho$ for all $x, y \in \operatorname{ker} \rho_{2}$. On the other hand, if $x \rho \in E_{S / \rho}$, then $x \in \operatorname{ker} \rho_{2}$. It follows that $E_{S / \rho}$ forms a rectangular band, therefore, $S / \rho$ is a rectangular group (Theorem 1.6(iv)). Finally, suppose by way of contradiction that $\rho$ is a matrix congruence on $S$, that is, $(a b a, a) \in \rho$ for all $a, b \in S$. Then $(a b a, a) \in \rho_{2}$ for all $a, b \in S$. Hence $S / \rho_{2}$ must be a trivial group. Thus $\rho_{2}=S \times S$, a contradiction from the assumption that $\rho_{2}$ is a non-universal congruence on $S$. Similarly, if $\rho$ is a group congruence, then $\rho_{1}$ is a group congruence (since $\rho \subseteq \rho_{1}$ ), so $S / \rho_{1}$ must be trivial. Hence $\rho_{1}$ is the universal relation, but this is impossible. Consequently, $\rho$ is a proper rectangular group congruence on $S$.

We have just seen that the intersection of a group congruence on a semigroup $S$ and a matrix congruence on $S$ is a rectangular group congruence on $S$. Conversely, the part (i) of the following theorem (together with Theorem 2.1) implies that any rectangular group congruence on $S$ can be expressed in this way.

Theorem 2.2 Let $\rho$ be a rectangular group congruence on a semigroup $S$ (and so $S / \rho=M \times G$, where $M$ is a rectangular band, $G$ is a group). Also, let $Q_{m}(m \in M)$ be the preimage of $\{m\} \times G$ by the canonical epimorphism $\rho^{\natural}$ from $S$ onto $S / \rho$, and put $N=\left\{s \in S: s \rho \in E_{M \times G}\right\}$. Moreover, denote by $v$ the matrix congruence on $S$, induced by the partition $\left\{Q_{m}: m \in M\right\}$ of $S$ (see the proof of “(i) $\Longrightarrow$ (ii)" in Theorem 2.1). Then:

(i) $\rho=v \cap \rho_{N}$;

(ii) $S / \rho \cong S / v \times S / \rho_{N}$.

If in addition $S$ is $E$-inversive, then:

(iii) $\forall m \in M\left[N \cap Q_{m} \triangleleft Q_{m}\right]$;

(iv) $\forall m \in M\left[S / \rho_{N} \cong Q_{m} / \rho_{\left(N \cap Q_{m}\right)}\right]$.

Proof Firstly, notice that $N$ is the preimage of $M \times\left\{1_{G}\right\}$ by $\rho^{\natural}$. Secondly, every $Q_{m}$ is a quasi dense subsemigroup of $S$ (see Result 1.4). Also, if $S$ is $E$-inversive, then each $Q_{m}$ is an $E$-inversive subsemigroup of $S$ (Result 1.7).

(i). Let $(a, b) \in \rho$ and $a \rho=(m, g)$, where $(m, g) \in M \times G$. Take $x=\left(m, g^{-1}\right)$, where $g^{-1}$ is a group inverse of $g$ in $G$. Then clearly $x a, x b \in N$ and so $(a, b) \in \rho_{N}$ (see Remark 1). Also, $a \rho=(m, g)=b \rho \in\{m\} \times G$. Hence $a, b \in Q_{m}$, so $(a, b) \in v$. 
Thus $(a, b) \in v \cap \rho_{N}$. Consequently, $\rho \subset v \cap \rho_{N}$. Conversely, let $(a, b) \in v \cap \rho_{N}$. Then $a \rho, b \rho \in\{m\} \times G\left(a \rho=\left(m, g_{1}\right), b \rho=\left(m, g_{2}\right)\right), x a, x b \in N$ for some $m \in M$ and $x \in Q_{n}$, where $n \in M$ (say $\left.x \rho=(n, g)\right)$, and so $(x a) \rho=\left(n m, g g_{1}\right)$. On the other hand, $(x a) \rho \in M \times\left\{1_{G}\right\}$. Hence $g_{1}=g^{-1}$. We may equally well show that $g_{2}=g^{-1}$. Thus $(a, b) \in \rho$. Consequently, $\rho=v \cap \rho_{N}$, as exactly required.

(ii). Indeed, $\rho=v \cap \rho_{N}$ by (i). Define the mapping $\phi: S / \rho \rightarrow S / v \times S / \rho_{N}$ by $(a \rho) \phi=\left(a v, a \rho_{N}\right)(a \in S)$. Clearly, $\phi$ is a monomorphism. We show that $\phi$ is surjective. Let $\left(a v, b \rho_{N}\right) \in S / v \times S / \rho_{N}$. Then $a \in Q_{m}$, where $m \in M$. Take any element $n \in N \cap Q_{m}$. Then

$$
((n b n) \rho) \phi=\left((n b n) v,(n b n) \rho_{N}\right)=\left(n v, b \rho_{N}\right)=\left(a v, b \rho_{N}\right) .
$$

(iii). Let $m \in M$. Put $N_{m}=N \cap Q_{m}$. Evidently, $N_{m}$ is a full, reflexive and closed subsemigroup of $Q_{m}$ (even if $S$ is an arbitrary semigroup). By Result 1.7, $N_{m}$ is dense in $Q_{m}$. Thus $N_{m} \triangleleft Q_{m}$.

(iv). Let $m \in M$. Define the map $\phi: Q_{m} / \rho_{N_{m}} \rightarrow S / \rho_{N}$ by $\left(a \rho_{N_{m}}\right) \phi=a \rho_{N}$ ( $a \in Q_{m}$ ). Clearly, $\phi$ is a well-defined homomorphism. Furthermore, if $a \in S$ and $n \in N_{m} \subseteq N$, then nan $\in Q_{m}$ and ( (nan) $\left.\rho_{N_{m}}\right) \phi=($ nan $) \rho_{N}=a \rho_{N}$. Thus $\phi$ is surjective. Finally, we show that $\phi$ is injective. Let $a, b \in Q_{m},\left(a \rho_{N_{m}}\right) \phi=\left(b \rho_{N_{m}}\right) \phi$. Then $(a, b) \in \rho_{N}$, so $a x, b x \in N$ for some $x \in S$. Hence for every $n \in N_{m} \subseteq N$, anx, bnx $\in N$. Thus $n($ anx $) n \in N \cap\left(Q_{m} N Q_{m}\right) \subseteq N \cap Q_{m}=N_{m}$ and similarly: $n(b n x) n \in N_{m}$. Since $n a, n b, n x n \in Q_{m}$, then $(n a, n b) \in \rho_{N_{m}}$, and so $(a, b) \in \rho_{N_{m}}$, because $n \in N_{m}=\operatorname{ker} \rho_{N_{m}}$.

Corollary 2.3 If the least group congruence exists on a semigroup $S$, then the relation $\psi \cap \sigma$ is the least rectangular group congruence on $S$. In particular, in any $E$-inversive semigroup, $\psi \cap \sigma$ is the least rectangular group congruence.

Remark 2 If $S$ is not $E$-inversive, then the least rectangular group congruence on a semigroup $S$ may not exist. Indeed, consider the additive semigroup of non-negative integers $\mathbb{N}$. It is well known that every group congruence on $\mathbb{N}$ is of the following form: $\rho_{n}=\{(k, l) \in \mathbb{N} \times \mathbb{N}: n \mid(k-l)\}(n>0)$. Further, since $\mathbb{N}$ has identity, then the least matrix congruence on $\mathbb{N}$ is the universal relation, so any rectangular group congruence on $\mathbb{N}$ is a group congruence (Theorem 2.2(i)). Consequently, $\mathbb{N}$ has no least rectangular group congruence.

Let $\mathcal{C}$ be a class of semigroups which is closed under homomorphic images. Note that if the least $\mathcal{C}$-congruence $\rho_{\mathcal{C}}$ on a semigroup $S$ exists, then the interval $\left[\rho_{\mathcal{C}}, S \times S\right.$ ] consists of all $\mathcal{C}$-congruences on $S$ and it is a complete sublattice of the complete lattice $\mathcal{C}(S)$ of congruences on $S$. Evidently, the class of all groups [rectangular bands] is closed under homomorphic images. Using Theorem 1.6(iv) one can prove without difficulty that the class of all rectangular groups has this property. Denote by $\theta$ the least rectangular group congruence on an $E$-inversive semigroup. In particular, the intervals $[\psi, S \times S],[\sigma, S \times S],[\theta, S \times S]$ consist of all matrix, group, rectangular group congruences on an $E$-inversive semigroup $S$, respectively, and they are complete sublattices of $\mathcal{C}(S)$. Denote them by $\mathcal{M C}(S), \mathcal{G C}(S), \mathcal{R G C}(S)$, respectively. Clearly, the direct product $\mathcal{M C}(S) \times \mathcal{G C}(S)$ is a complete sublattice of $\mathcal{C}(S) \times \mathcal{C}(S)$ (see [3, p. 37]). 
For terminology and elementary facts about lattices the reader is referred to the book [10, Sect. I.2]. The following simple result will be useful (see Lemma I.2.8 and Exercise I.2.15(iii) in [10]).

Result 2.4 If $\phi$ is an order isomorphism of a lattice $L$ onto a lattice $M$, then $\phi$ is a lattice isomorphism. Moreover, every lattice isomorphism of complete lattices is a complete lattice isomorphism.

We show that each rectangular group congruence on an $E$-inversive semigroup can be expressed as the unique intersection of a group and a matrix congruence.

Theorem 2.5 Every rectangular group congruence on an E-inversive semigroup $S$ is of the form $v \cap \rho_{N}$, where $v$ is a matrix congruence on $S, N \triangleleft S$, and this expression is unique.

Moreover, there exists an inclusion-preserving bijection $\phi$ between the complete lattice $\mathcal{M C}(S) \times \mathcal{G C}(S)$ and the complete lattice $\mathcal{R G C}(S)$. In fact, $\phi$ is defined by:

$$
\left(v, \rho_{N}\right) \phi=v \cap \rho_{N}
$$

for every $\left(v, \rho_{N}\right) \in \mathcal{M C}(S) \times \mathcal{G C}(S)$. Furthermore, $\phi^{-1}$ is an inclusion-preserving bijection (by the proof of Theorem 2.2(i)), so $\phi$ is an order isomorphism of the complete lattice $\mathcal{M C}(S) \times \mathcal{G C}(S)$ onto the complete lattice $\mathcal{R G C}(S)$. Consequently, $\phi$ is a complete lattice isomorphism between the lattices $\mathcal{M C}(S) \times \mathcal{G C}(S)$ and $\mathcal{R G C}(S)$, respectively.

Proof Let $\rho$ be a rectangular group congruence on $S$. Then $\rho$ is the intersection of some matrix and some group congruence on $S$ (Theorem 2.2(i)). Next, suppose that $\rho=v_{1} \cap \rho_{N_{1}}=v_{2} \cap \rho_{N_{2}}$, where $v_{i}$ is a matrix congruence on $S, N_{i} \triangleleft S(i=1,2)$. Let $(a, b) \in v_{1}$. Since $v_{1} \cap v_{2}$ is a matrix congruence on $S$, then there are idempotents $e, f$ of $S$ such that $(a, e) \in v_{1} \cap v_{2},(e, f) \in \rho_{N_{1}},(f, b) \in v_{1} \cap v_{2}$ (Result 1.7), so $(e, f) \in v_{1} \cap \rho_{N_{1}}=v_{2} \cap \rho_{N_{2}} \subseteq v_{2}$. Hence $(a, b) \in v_{2}$, i.e., $v_{1} \subset v_{2}$. We may equally well show the opposite inclusion. Put $v_{1}=v_{2}=v$, so that $\rho=v \cap \rho_{N_{1}}=v \cap \rho_{N_{2}}$. If $(a, b) \in \rho_{N_{1}}$, then $(a a b, a b b) \in v \cap \rho_{N_{1}} \subset \rho_{N_{2}}$, so $(a, b) \in \rho_{N_{2}}$ (by cancellation). Hence $\rho_{N_{1}} \subset \rho_{N_{2}}$. By symmetry, $\rho_{N_{2}} \subset \rho_{N_{1}}$. Thus $\rho_{N_{1}}=\rho_{N_{2}}$, as exactly required.

The second part of the theorem follows directly from the above considerations and Result 2.4.

Finally, from Result 1.7 we obtain the following theorem.

Theorem 2.6 Every rectangular group congruence on an E-inversive semigroup $S$ is uniquely determined by its kernel and trace.

Proof Let $\rho_{1}, \rho_{2} \in \mathcal{G C}(S), v_{1}, v_{2} \in \mathcal{M C}(S)$ be such that $\operatorname{ker}\left(v_{1} \cap \rho_{1}\right) \subset \operatorname{ker}\left(v_{2} \cap \rho_{2}\right)$ and $\operatorname{tr}\left(v_{1} \cap \rho_{1}\right) \subset \operatorname{tr}\left(v_{2} \cap \rho_{2}\right)$. Then

$$
\operatorname{ker}\left(v_{1} \cap \rho_{1}\right)=\operatorname{ker} v_{1} \cap \operatorname{ker} \rho_{1}=S \cap \operatorname{ker} \rho_{1}=\operatorname{ker} \rho_{1} \subset \operatorname{ker} \rho_{2} .
$$

In the light of Lemma 1.9, $\rho_{1} \subset \rho_{2}$. Similarly, we obtain that $\operatorname{tr} v_{1} \subset \operatorname{tr} v_{2}$. Hence $v_{1} \subset v_{2}$ (this follows from the proof of Result 1.7, see [1]). Thus $v_{1} \cap \rho_{1} \subset v_{2} \cap \rho_{2}$. This implies the thesis of the theorem. 
Open Access This article is distributed under the terms of the Creative Commons Attribution License which permits any use, distribution, and reproduction in any medium, provided the original author(s) and the source are credited.

\section{References}

1. Gigoń, R.S.: Some results on E-inversive semigroups. Quasigroups Relat. Syst. 20, 53-60 (2012)

2. Gigoń, R.S.: Congruences and group congruences on a semigroup. Semigroup Forum. doi:10.1007/ s00233-012-9425-z

3. Howie, J.M.: Fundamentals of Semigroup Theory. Oxford University Press, New York (1995)

4. Howie, J.M.: An Introduction to Semigroup Theory. Academic Press, London (1984)

5. Masat, F.E.: Right group congruences on a semigroup. Proc. Am. Math. Soc. 50, 107-114 (1975)

6. Masat, F.E.: Right group and group congruences on a regular semigroups. Duke Math. J. 40, 393-402 (1973)

7. Mitsch, H., Petrich, M.: Basic properties on E-inversive semigroups. Commun. Algebra 28, 5169$5182(2000)$

8. Mitsch, H., Petrich, M.: Restricting idempotents in $E$-inversive semigroups. Acta Sci. Math. (Szeged) 67, 555-570 (2001)

9. Petrich, M.: The maximal matrix decomposition of a semigroup. Port. Math. 25, 15-33 (1966)

10. Petrich, M.: Inverse Semigroups. Wiley, New York (1984) 